\title{
Microstructural Characterization of an Irradiated 304 Stainless Steel
}

\author{
Yimeng Chen, Emmanuelle A. Marquis
}

Department of Materials Science and Engineering, University of Michigan, Ann Arbor, Michigan 48109-2136, USA

The irradiation behavior of 304 steels has been investigated for decades. Yet, it remains unclear how dislocation loop and cluster density vary with alloy composition, irradiation conditions and postannealing treatments, and most importantly how they affect mechanical properties. The post-annealing treatments are often considered to remediate the loss in mechanical properties, i.e. strength and stress corrosion cracking initiation. Mechanical property models used to predict the strength evolution of 304 during irradiation are often based on a simple linear summation of the contributions from the different defect populations, in particular loops and clusters. This approach assumes that the two populations of defects would be spatially independent.

In many studies the microstructures of irradiated 304 have been investigated by transmission electron microscopy (TEM) to study the dislocation loop density and atom probe tomography (APT) to reveal the distribution of solutes. After neutron irradiation in commercially pure (CP) 304, Toyama et al. reported the formation of $10 \mathrm{~nm}$ diameter Ni-Si clusters [1]. Because a much lower dislocation density than that of the clusters was observed, the authors concluded that the clusters form independently from the loops. In different proton irradiated CP 304, Jiao et al. also reported the presence of smaller clusters with size, number density and morphology dependent on the alloy Si concentration [2]. The alloys were compared based on cluster sizes and number densities that were analyzed by the maximum separation method [3] irrespective of the actual cluster morphology.

APT is probably the only technique qualified for detailed microstructural characterization for small clusters and segregations on loops. However, there is no universal method for analyzing such microstructure in 304 which would allow direct comparisons with values reported in the literature or comparisons between different alloys or different processing conditions. It is important to note that the output of the maximum separation distance algorithm is extremely sensitive to the choice of the parameters that serve as input. This is problematic for description of material microstructure with diffuse interfaces and features with compositions that are close to that of the matrix. Therefore the aim of this study is to develop a robust method that would provide quantitative spatial and chemical description of these irradiated 304 microstructures.

A CP 304 stainless steel was implanted using $2 \mathrm{MeV}$ protons at $360^{\circ} \mathrm{C}$ to $10 \mathrm{dpa}$ and a post-annealing treatment was carried out at $500^{\circ} \mathrm{C}$ for 1 hour. To study the evolution of the microstructure, APT specimens from as-irradiated and annealed materials were prepared by lift-out and ion beam milling. TEM observations of the needle-shaped specimens were conducted before and after APT analysis to provide independent visualization of the defects present in the structure as well as measurement of the scaling parameters (specimen apex radius and shank angle).

The TEM images in Figure 1 show the presence of high density of dislocation loops and precipitates in the as-irradiated sample. The APT reconstructions reveal a complex distribution of solute atoms with $\mathrm{Ni}$ and $\mathrm{Si}$ clusters, $\mathrm{Cu}$ clusters and $\mathrm{Si}, \mathrm{Ni}, \mathrm{P}$ segregation at line features, assumed to be dislocation loops. 
The spatial distribution of the clusters is not independent and it can be seen from Figure 2 that the Ni-Si clusters are correlated with the location of dislocations and the $\mathrm{Cu}$ clusters are often associated with NiSi clusters. The challenge resides in quantitatively describe these features.

Quantitative methods for analyzing such complex and convoluted microstructures will be discussed. The ultimate objective is to understand the microstructural evolution taking place during irradiation and postirradiation annealing.

\section{References:}

[1] Toyama et al, Jounal of Nuclear Materials 418 (2011) p62-68

[2] Z. Jiao and G.S.Was, Acta Materialia 59(2011) p1220-1238

[3] D. Vaumousse et al, Ultramicroscopy 95 (2003), p215-221

[4] The work is funded by EPRI and materials were provided by Dr. Z. Jiao and Prof. G.S. Was at the University of Michigan.
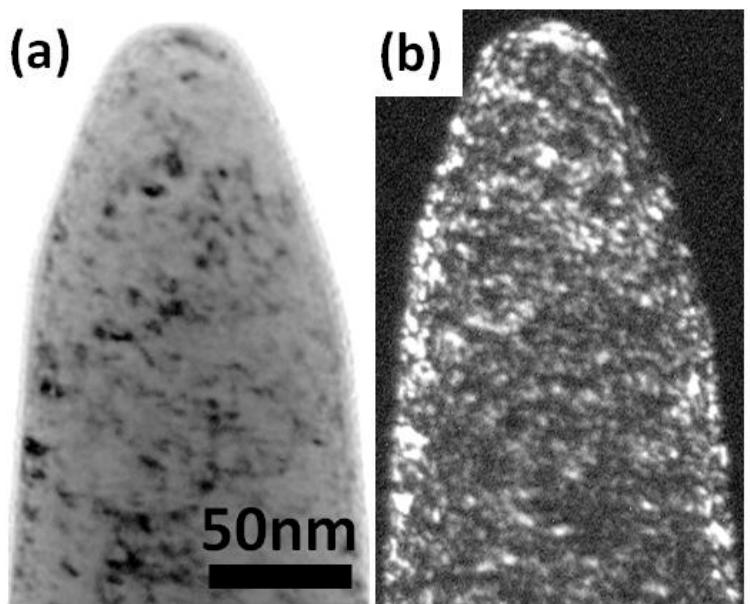

Figure 1. (a) and (b) are bright and dark field TEM images taken from a 304 tip specimen in the asirradiated condition.

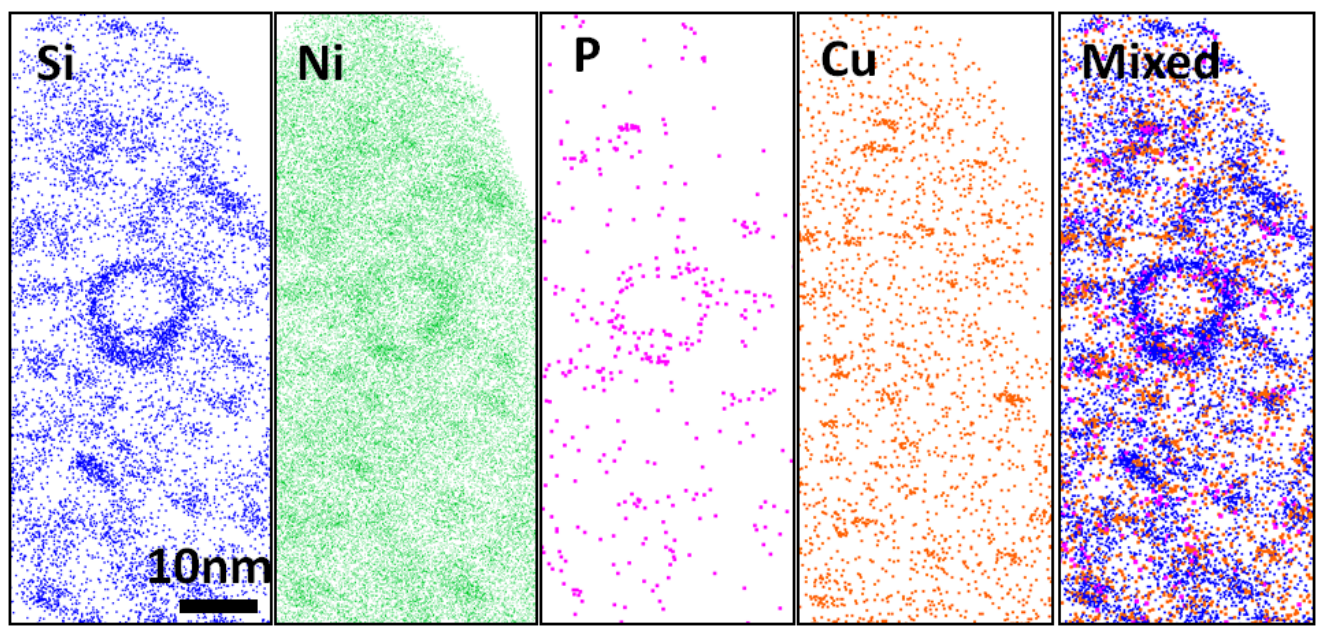

Figure 2. 5nm sliced APT reconstruction maps showing distribution of $\mathrm{Si}, \mathrm{Ni}, \mathrm{P}$ and $\mathrm{Cu}$ in 304 in the asirradiated condition. 